\title{
Enhancing Content-based Image Retrieval using Moving K-Means Clustering Algorithm
}

\author{
Shefalli \\ Research Scholar \\ Yadavindra College of Engineering, \\ Guru Kashi Campus, Punjabi University, \\ Talwandi Sabo, Punjab, India
}

\author{
Balkrishan Jindal \\ Assistant Professor \\ Yadavindra College of Engineering, \\ Guru Kashi Campus, Punjabi University, \\ Talwandi Sabo, Punjab, India
}

\begin{abstract}
In this paper, new content-based image retrieval (CBIR) system based on color and shape feature combining with clustering concept that considers the similarities among images in database is proposed. Firstly, the color space of an image is quantized with 128 bin quantization and then color histogram is extracted from the quantized image. Secondly, the shape feature is extracted using horizontal, vertical, diagonal and anti-diagonal mask obtained by rotation of Prewitt mask. Thirdly, the moving k-means clustering algorithm is used to cluster the database images. The proposed method is tested on Wang database. Experimental results show that proposed method is more efficient and effective in image retrieving from database than existing methods.
\end{abstract}

\section{Keywords}

CBIR, clustering, color space, color histogram, Prewitt mask

\section{INTRODUCTION}

Today there is continuously increasing digital images collections. Daily large numbers of images are produced, so a proper organization of images is required for fast and better retrieval. For this purposes image retrieval systems are designed [1]. The image search becomes important due to popularity of various image search engines [2]. Image retrieval is process of retrieving image from the database of images. It is an extension of information retrieval where images are retrieved.

Text based image retrieval used text as the base by which images are retrieved from databases. Images were stored along with keywords prepared by an annotator and in future to retrieve that image one has to use that keyword. The main advantage of this technique is that there are well established methods for that and easy to understand but there exist difficulties, when size of database is large and for that vast amount of labor is required to manually annotate the images. The other difficulties are language and culture difference due to which same image is usually text out by many different ways and mistakes such as spelling error or spell difference gives different results $[1,3]$. To overcome these problems many techniques in CBIR have been developed and many CBIR systems have been built.

Content-based image retrieval is also known as query by image content (QBIC) and content-based visual information retrieval (CBVIR). The most common low level features of an image are color, texture and shape. These features are extracted automatically from the image. It is based on Query by example where query is an image from which features are extracted and it is compared with features of database images and according to that relevant images are retrieved [4].
Different methods are used to represent each feature each with advantages and disadvantages [5]. According to requirement and application best method can be selected out of these. CBIR systems have large number of applications like crime prevention, education and training, military application, medical diagnosis and fashion and interior design [6]. Some of the existing popular systems are flexible image retrieval engine (FIRE) [7] and photobook [8].

\section{RELATED WORK}

In 2010, [2] Chakravarti and Meng have proposed color histogram technique for image retrieval. This technique is efficient and easy to process. Daisy et al. [3] have proposed content based image retrieval using texture and shape feature. Gabor filter combined with morphological closing operation has been used to extract texture feature. Shape feature is extracted by using Fourier Descriptor. Buch et al. [4] have used color and texture feature for image retrieval. In this paper, color moment is used to extract color feature and wavelet and Gabor texture for texture feature. Adnan et al. [5] have explained image retrieval system architecture. In this paper, number of objects in an image, shape of objects, location of objects and geometrical parameters are considered as basis of indexing and retrieval. Pujari et al. [6] have explained content based image retrieval by using color and shape feature. It introduced Hue, Saturation and Value (HSV) color space and Lab color space techniques to capture the shape and color feature of the image. Deselaers et al. [7] have explained Flexible Image Retrieval Engine (FIRE) image retrieval system. This system is highly extensible offers wide varieties of features and distance functions. According to requirement a weighed combination of features can be used. Petland et al. [8] have described the Photobook system which is used for browsing and searching images. This system is quite flexible that allows text based as well as content based retrieval. This system provides an interactive interface for the user. In [10] Anantharatnasamy et al. have used color, texture and shape features in fusion. It used color histogram with 24 bin quantization to represent color feature. Grey Level Cooccurrence Matrix to represent texture feature and combination of chain method, area and horizontal to extract shape feature. Acharjya et al. [11] have described shape detection of an image. It explained Prewitt's mask to detect an edge image. Liu and $\mathrm{Yu}$ [12] have explained k-means clustering algorithm in image retrieval system. The k-means clustering algorithm considered the similarities among images in the database and make retrieval fast and more accurate as compare to system without clustering.

After literature review, it has been concluded that many of the existing content-based image retrieval (CBIR) techniques have problem of high computation time due to comparison of 
query image with all the database images. The another problem is related with accuracy i.e. accuracy of some of the systems is not very good.

\section{PROPOSED METHOD}

The proposed method uses the moving k-means clustering algorithm to cluster the database images. First collection of images is taken. Then color space of an image is quantized with 128 bin quantization and color histogram is extracted from the quantized image. Secondly, the shape feature is extracted using horizontal, vertical, diagonal and anti-diagonal mask obtained by rotation of Prewitt mask. After extracting color and shape feature from all images, a feature database of images is prepared. The moving $\mathrm{k}$-means clustering algorithm is applied to clustering the feature database. On the other side query image is given as input and similar features with same technique are extracted from the image. These features are compared with each cluster and find the closest cluster to query image using euclidean distance. After selection of cluster required number of images are displayed from that cluster as resultant images. Figure 1 shows block diagram of image retrieval system with proposed method.

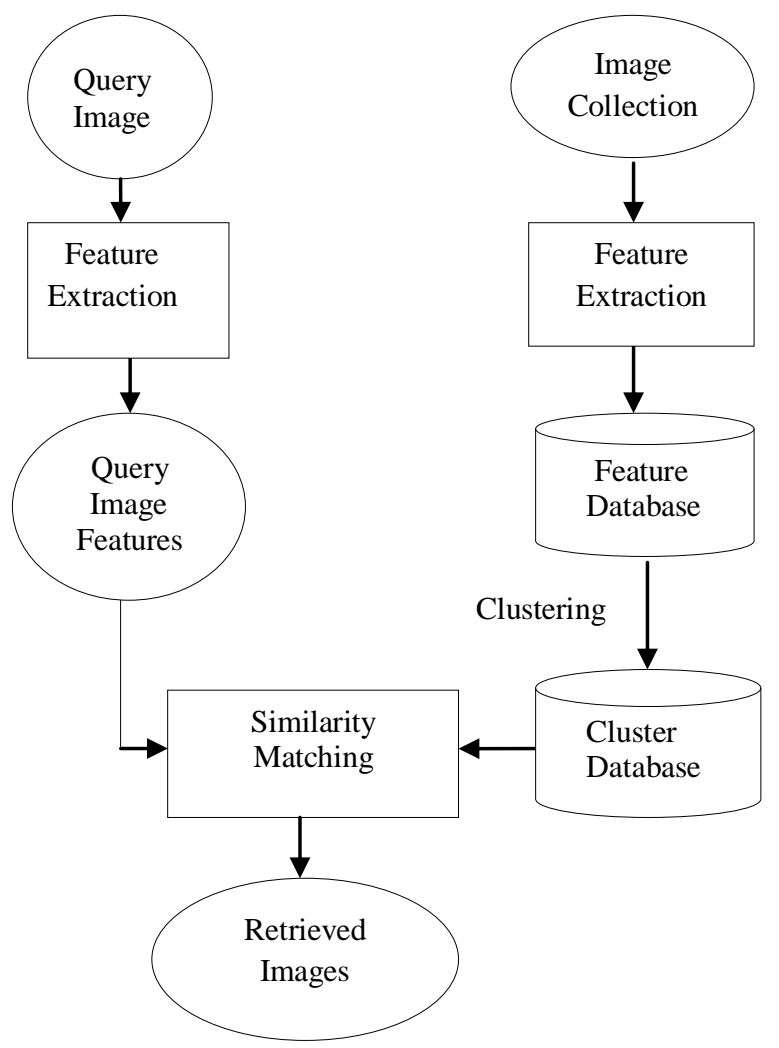

Figure 1: Block diagram of image retrieval system with proposed method

\subsection{Feature extraction}

\subsubsection{Color feature extraction}

In this work, color histogram technique has been used to extract color feature. The main issue regarding color feature are choice of color space, quantization of color space and color descriptor selection.

\subsubsection{Color space selection}

In this work, Hue, Max, Min and Diff (HMMD) color space has been used. The images are present in database in Red,
Green and Blue (RGB) format which is converted into HMMD color space. The Hue (0 to 360 degrees) component represent color in its pure form i.e. red, blue or green. Max and Min represent the maximum and minimum among the R, $\mathrm{G}$ and $\mathrm{B}$ values respectively. Diff component is defined as the difference between max and min value. There is another component Sum that is defined as average of max and min value. Out of five, three components $\{\mathrm{H}, \mathrm{Max}, \mathrm{Min}\}$ or $\{\mathrm{H}$, Diff, Sum $\}$ are sufficient to form HMMD color space. Max $(\mathrm{M}=[0,1])$ represent how much black color is present i.e. represent shade or blackness. Min $(\mathrm{M}=[0,1])$ represent how much white color is present i.e. tint or whiteness. Diff $(D=[0$, 1]) specifies how much a color is close to pure color i.e. tone or colorfulness. Sum $(=[0,1])$ specify brightness of color. The HMMD color space [9] is effective and better than HSV color space.

\subsubsection{Quantization of color space}

Quantization is a process in which colors in a color space are reduced by putting the similar colors in a single bin. It helps to reduce the computation time and comparison time of colors. Without quantization, to find similarity between two images one has to compare 16777216 colors that will take lot of time. In this work, 128 bin quantization of HMMD color space has been used [9]. In quntization processs first quantization table is formed and with the help of quantization table quantized image is formed. With quantization, the bins of two images are compared to find the similarity between images.

\subsubsection{Color descriptor selection}

In this work, Color histogram is used to represent color feature. The histogram of an image represent how many pixels are present at particular grey level [10].

\subsubsection{Shape feature extraction}

For shape feature first edges of the image are find out. For that purpose at beginning a colored image is chosen. This image is divided into four parts and is converted into gray scale image. A gray scale image is combination of two colors i.e. black and white. These colors carry information regarding the intensity. Black color represent the low intensity and white color represent the high intensity. Variations in this intensity levels forms edges. In this case edges are detected from four directions.To detect edges, masks are applied to gray scale image. In this work, horizontal, vertical, diagonal and antidiagonal mask has been used. These masks can be obtained by rotating the Prewitt's mask [11]. These masks are in horizontal, vertical, diagonal and anti-diagonal directions. After applying all masks different sub images are find out in all directions.

Prewitt Mask

\begin{tabular}{|r|r|r|}
\hline 1 & 1 & 1 \\
\hline 0 & 0 & 0 \\
\hline-1 & -1 & -1 \\
\hline
\end{tabular}

Masks used in proposed method

\begin{tabular}{|l|l|l|}
\hline 1 & 1 & 1 \\
\hline 0 & 0 & 0 \\
\hline-1 & -1 & -1 \\
\hline \multicolumn{3}{|l|}{} \\
\hline 1 & 0 & -1 \\
\hline 1 & 0 & -1 \\
\hline 1 & 0 & -1 \\
\hline
\end{tabular}

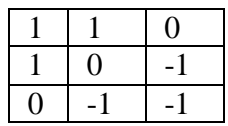

\begin{tabular}{|c|c|c|}
\hline 0 & 1 & 1 \\
\hline-1 & 0 & 1 \\
\hline-1 & -1 & 0 \\
\hline
\end{tabular}




\subsubsection{Clustering of database}

Clustering is to group data elements of a data set into clusters with some similarity criteria. In CBIR systems similarity among the images of database is not considered only the similarity between query image and database images is considered for retrieval [12]. In this problem of high computation time exists due to comparison of query image features with features of all images in database. To reduce computation time clustering is used that considers similarity among the images of database. After clustering there is no need to compare query image with all database images that reduces the computation time. It also helps to improve accuracy. The k-means clustering algorithm is very simple and easy to implement. The problem with this method is that the clusters generate are not of good quality and time taken to generate clusters is very high. The moving k-means clustering algorithm generates good quality clusters and the time taken to generate cluster is less than $\mathrm{k}$ means algorithm.

In moving k-means clustering algorithm, first user have to give input the number of desired clusters. The data set is randomly divided into no. of desired clusters. In each cluster the middle point is considered as the centroid of cluster. The distance between each data point to all the initial centroid is calculated and data point is assigned to the cluster with closest centroid. During this a record of the cluster to which data item is assigned and distance of the data item to that cluster is kept. Recalculate the centroids of the cluster. Again for each data point compute its distance from the centroid of the present nearest cluster and if this distance is less than or equal to the present nearest distance, the data point stays in the same cluster else distance of the data item is calculated form all the centroids. This process continuous until the convergence criteria is not satisfied.

\section{PROPOSED ALGORITHM}

The various steps used in proposed algorithm works as follows:

Step-1: First collect the images from which one wants to retrieve the images. Collection depends upon the field for which image retrieval system is designed.

Step-2: Read the images from database one by one and extract the color and shape features from the image. Then prepare the feature database that contains the features of all images.

Step-3: Apply moving k-means clustering algorithm to the feature database.

Step-4: Read the query image and extract the same feature with same method as used for database images.

Step-5: Compute similarity between query image features and clustered database by Euclidean distance and find the cluster closest to query image.

Step-6: Display the required no. of images from the selected cluster.

\section{EXPERIMENTAL RESULTS}

In this section, results of proposed method are presented and discussed. The proposed method is implemented in MATLAB. The Wang database [13] is used for evaluation which is a subset of Corel database with total 1000 images. All images are classified into 10 categories like Africans, Beaches, Architecture, Buses, Dinosaurs, Elephants, Flowers, Horses, Mountains and Foods with 100 images in each category. All images in database are colored images and of size $384 \times 256$ or $256 \times 384$ pixels. The evaluation of retrieval results can be easily made due to this classification. Figure 2 shows the sample of 10 images of 10 different category of Wang database [13]. The images in the same row belongs to same category.

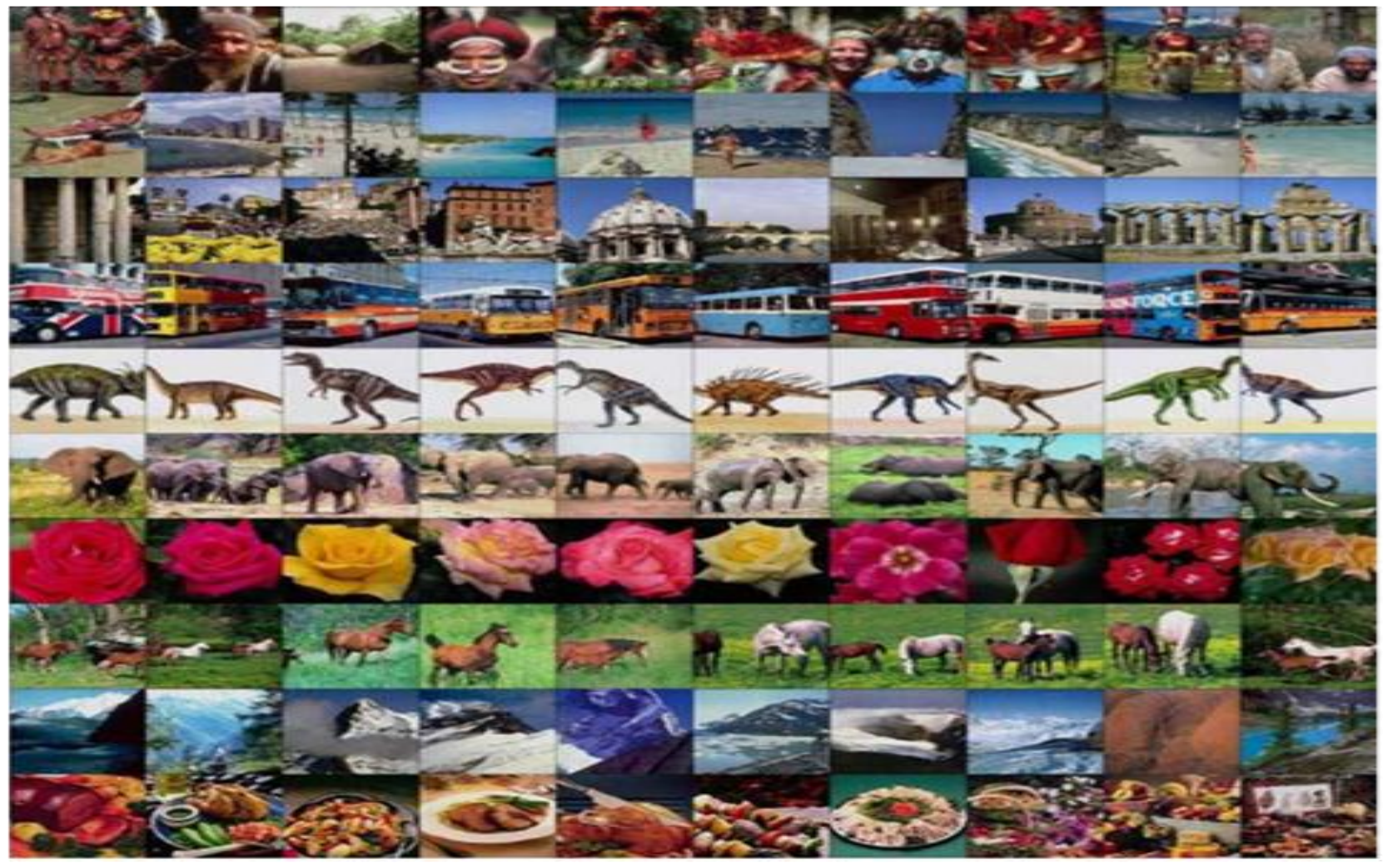

Figure 2: Sample of 10 images of 10 different category of Wang database 
The performance of the proposed method is evaluated with standard parameters, i.e., precision and recall [14].

$$
\begin{aligned}
& \text { Precision } \\
& =\frac{\text { Number of relevant images retrieved }}{\text { Total number of images retrieved }}
\end{aligned}
$$

Recall

$$
=\frac{\text { Number of relevant images retrieved }}{\text { Total number of relevant images in database }}
$$

The experiment was carried out by providing a query image from each category of database images. The precision and recall of each query image is computed by setting the number of output images as 16. The total no. of images in database for each individual category are 100 but here the required images are 16 so total no of relevant images in database is also considered as 16 for recall.

Figure 3-Figure 5 shows the retrieval results of bus, elephant and flowers query respectively with proposed method. The upper left image is query image and right part is the retrieved images. Due to shortage of length of paper results of only three categories has shown.

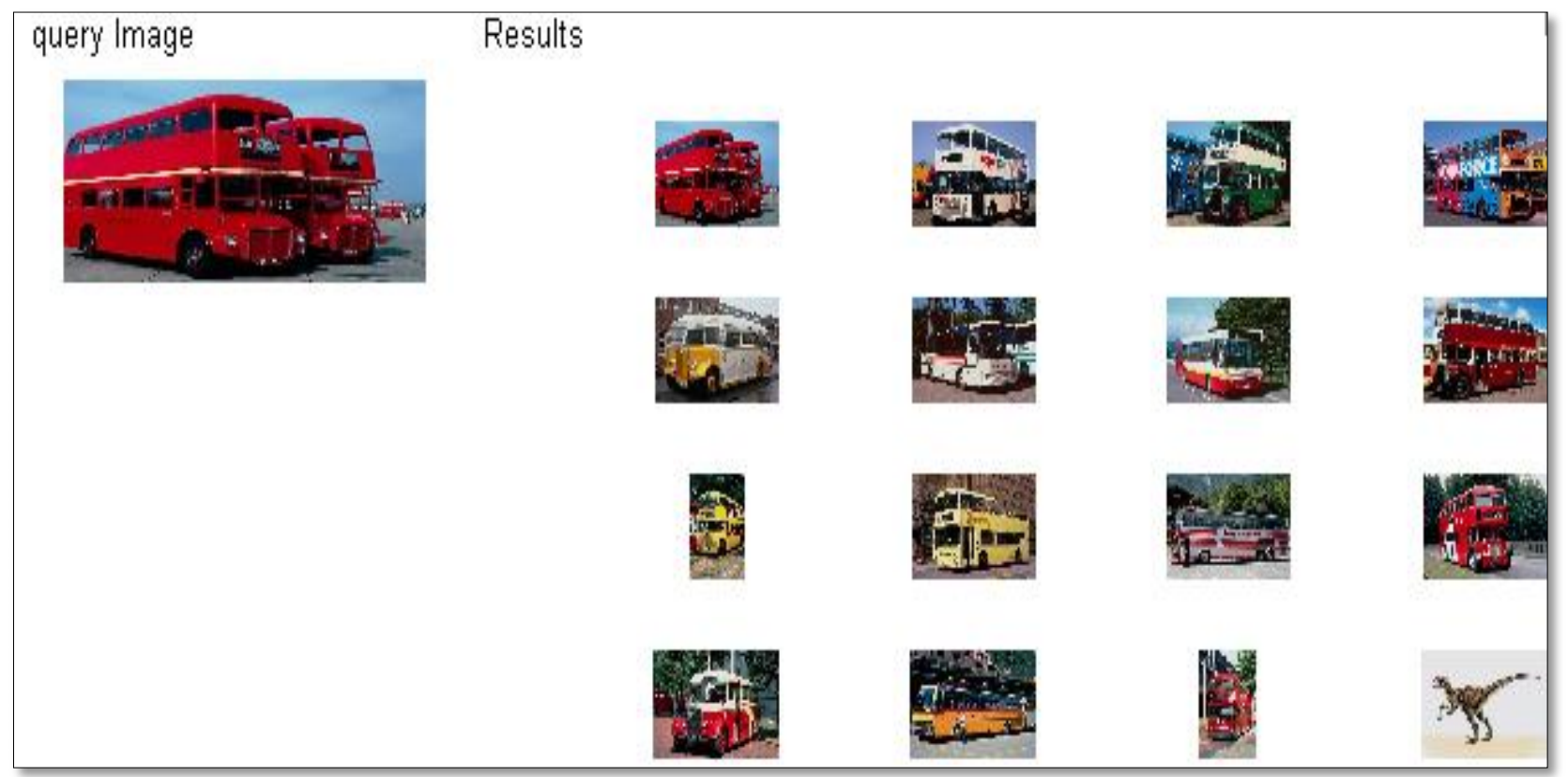

Figure 3: Retrieval results for bus query

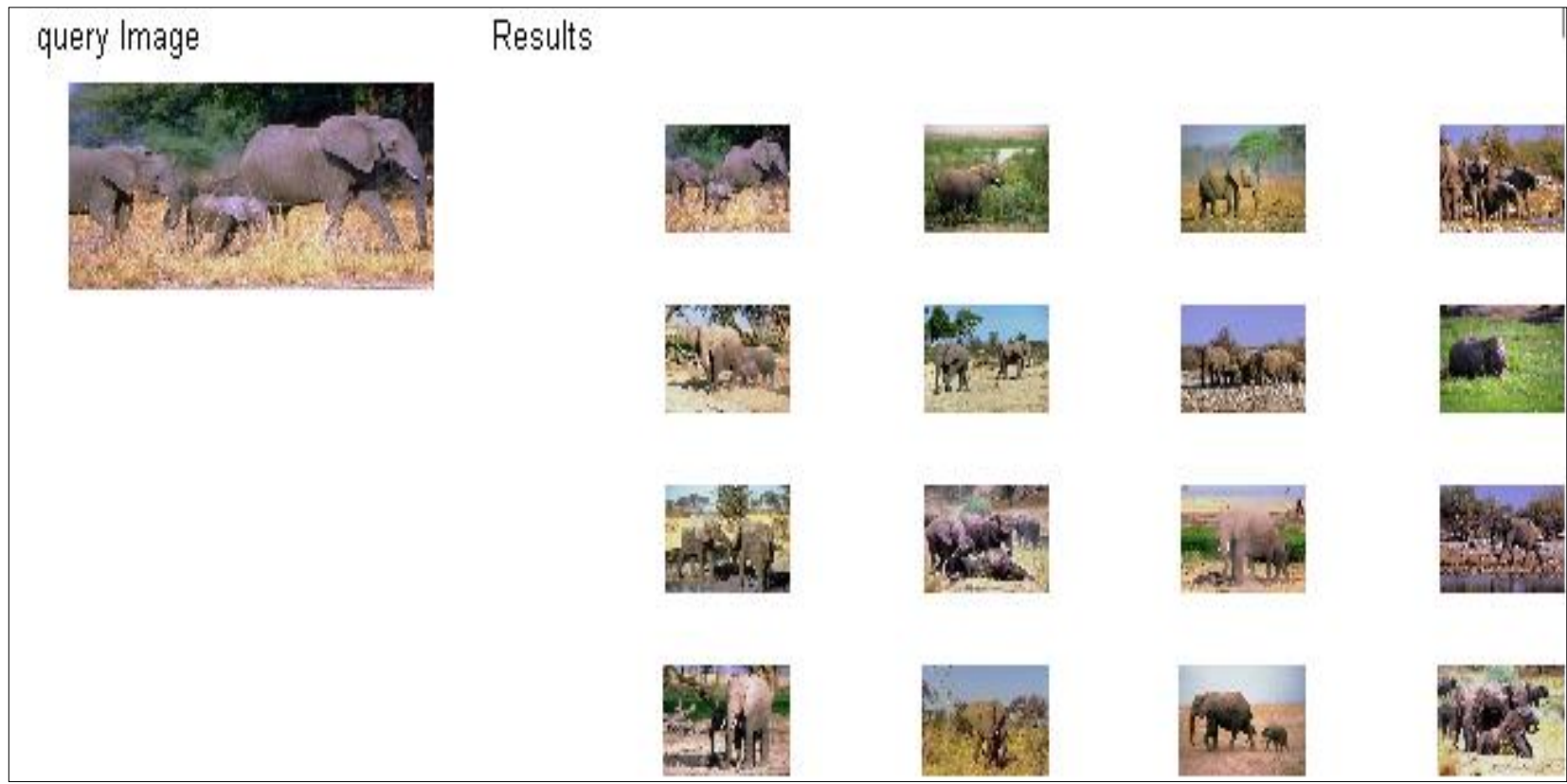

Figure 4: Retrieval results for elephant query 


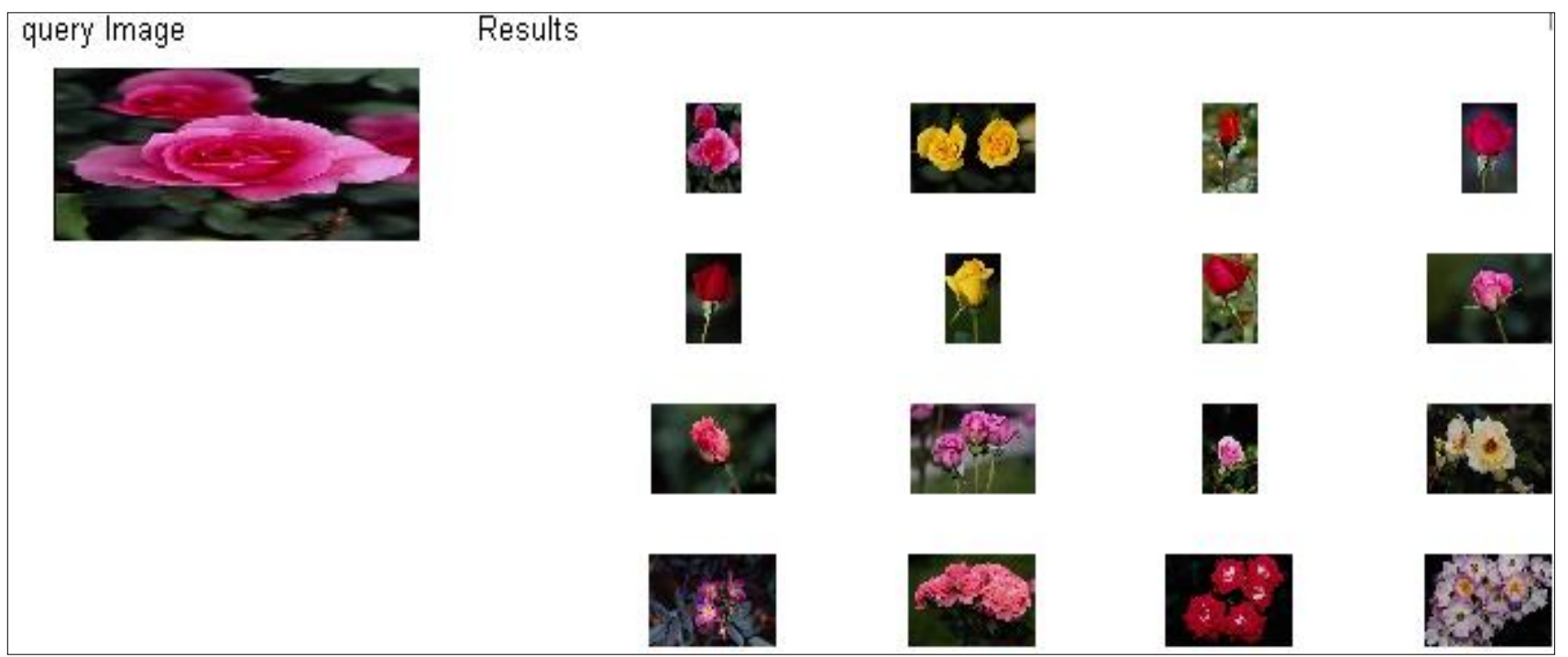

Figure 5: Retrieval results for flower query

To compare the performance of proposed method with Lande et al [14], each image is selected as query from each category i.e. Africans, Beaches, Architecture, Buses, Dinosaurs, Elephants, Flowers, Horses, Mountains and Foods and setting the number of output images to 20. Finally, the average precision and average recall of each category (100 images in each category) is computed. The total no. of images in database for each individual category are 100 but here the required images are 20 so total no of relevant images in database is also considered as 20 for recall. The average results of each individual category of proposed method and Lande et al [14] are compared and shown in Table 1.

Table 1 concluded that proposed method outperforms the method proposed by Lande et al both in term of average precision as well as average recall. The average precision category wise have improved for seven categories except for dinosaurs, horses and flowers category. Similarly the average recall category wise have improved for seven categories except for dinosaurs, flowers and horses category.

Table 1: Comparison of proposed method in terms of average precision and average recall using 10 indiviual category with Lande et al [14].

\begin{tabular}{|l|l|l|l|l|l|}
\hline \multirow{2}{*}{ Sr. No. } & \multirow{2}{*}{ Image Category } & \multirow{2}{*}{ Average Precision } & \multicolumn{2}{c|}{ Average Recall } \\
\cline { 3 - 6 } & & Lande et al [14] & Proposed Method & Lande et al [14] & Proposed Method \\
\hline 1 & Africnas & 0.75 & 0.80 & 0.75 & 0.80 \\
\hline 2 & Beaches & 0.55 & 0.79 & 0.55 & 0.79 \\
\hline 3 & Arcitecture & 0.73 & 0.82 & 0.73 & 0.82 \\
\hline 4 & Buses & 0.56 & 0.70 & 0.56 & 0.70 \\
\hline 5 & Dinosaurs & 0.99 & 0.90 & 0.99 & 0.90 \\
\hline 6 & Elephants & 0.75 & 0.85 & 0.75 & 0.85 \\
\hline 7 & Flowers & 0.85 & 0.75 & 0.85 & 0.75 \\
\hline 8 & Horses & 0.90 & 0.85 & 0.90 & 0.85 \\
\hline 9 & Mountains & 0.45 & 0.80 & 0.45 & 0.80 \\
\hline 10 & Foods & 0.75 & 0.76 & 0.75 & 0.76 \\
\hline
\end{tabular}


Figure 6 shows the overall average precision of proposed method and Lande et al [14]. Overall average precision is average of precision of all category. Overall average precision for proposed method is 0.802 and for Lande et al is 0.728 . It is concluded from figure 6 that proposed method is better than Lande et al in terms of overall average precision.

Figure 7 shows the overall average recall of proposed method and Lande et al [14]. Overall average recall is average of recall of all category. Overall average recall for proposed method is 0.802 and for Lande et al is 0.728 . It is concluded from figure 7 that proposed method is better than Lande et al in terms of overall average recall.

Figure 8 shows the comparison on the basis of average retrieval time. The average response time for proposed method is 0.95 and for Lande et al is 3.25. Figure concluded that average response time for a query in proposed method is very less as compare to Lande method.

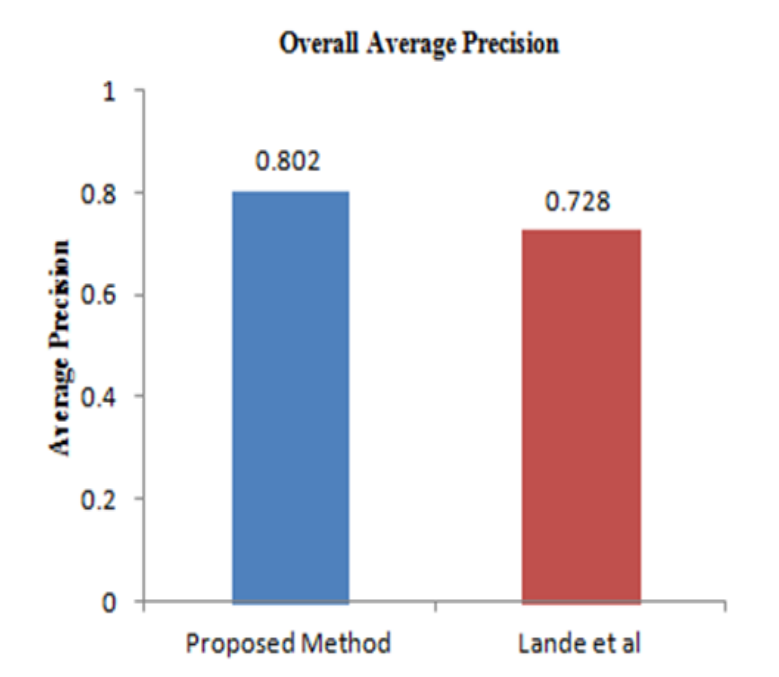

Figure 6: Comparison based on overall average precision

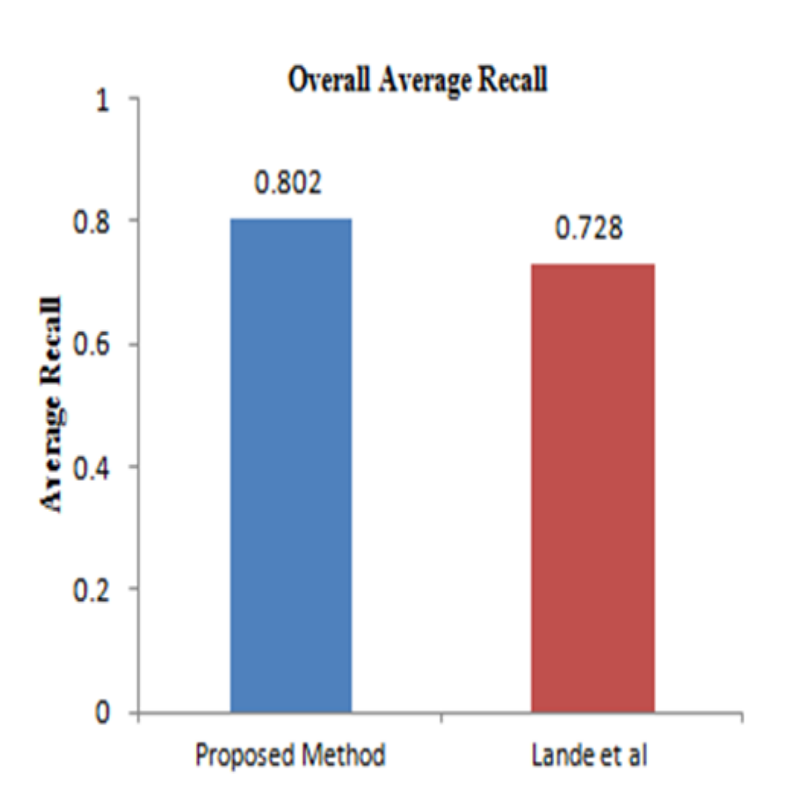

Figure 7: Comparison based on overall average recall

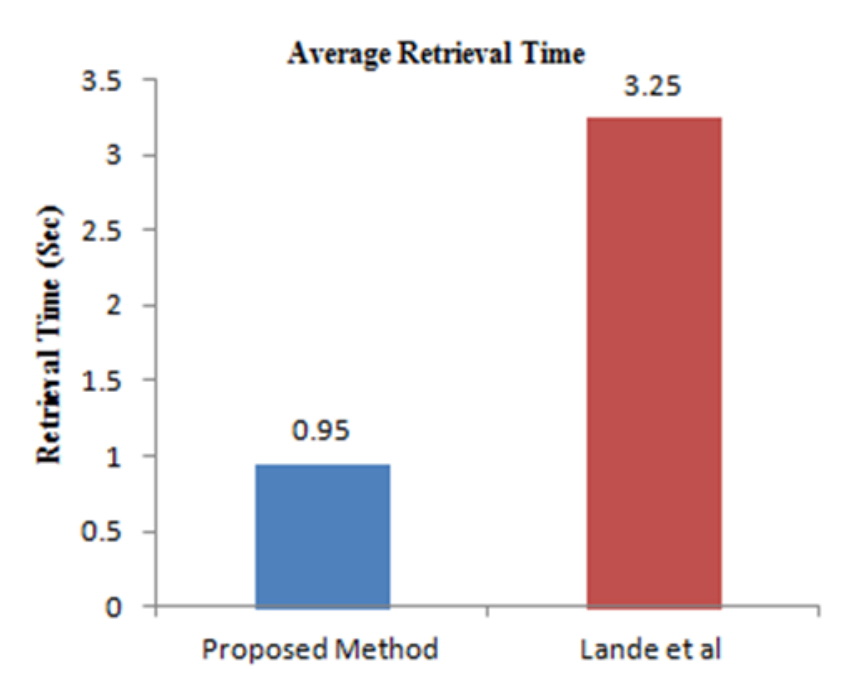

Figure 8: Comparison based on average retrieval time

\section{CONCLUSION}

In this work, an attempt has been made to retrieve the similar images from the database based on content based image retrieval method by supplying a query image. This method uses the combination of histogram color feature, four mask shape feature in four directions and moving k-means clustering algorithm that considers the similarities among images in database. Experimental results have shown that the proposed method is more effective and efficient than method proposed by Lande et al. In the future, the proposed image retrieval method is to be combined with the concept of relevance feedback to further improve the performance.

\section{REFERENCES}

[1] Rui, Y., Huang, T. S. and Chang, S. F., "Image Retrieval: Current Techniques, Promising Directions and Open Issues," vol. 4, pp. 33-62, 1 March, 1333.

[2] Chakravarti, R. and Meng, X., "A Study of Color Histogram Based Image Retrieval," Sixth International Conference on Information Technology: New Generations (ITNG'03), Las Vegas, pp. 1323-1326, 2523 April, 2003.

[3] Daisy, M.M.H., TamilSelvi, S. and GinuMol, J., "Combined texture and Shape Features for Content Based Image Retrieval," International Conference on Circuits, Power and Computing Technologies [ICCPCT2013], Nagercoil, pp. 912-916, 20-21 March, 2013.

[4] Buch, P.P., Vaghasia, M.V. and Machchhar, S.M, "Comparative analysis of content based image retrieval using both color and texture," International Conference on current trends in technology, Ahmadabad, Gujarat, pp. 1-4, 6-4 December, 2007.Sannella, M. J. 1994 Constraint Satisfaction and Debugging for Interactive User Interfaces. Doctoral Thesis. UMI Order Number: UMI Order No. GAX95-09398., University of Washington.

[5] Adnan, A., Gul, S., Ali, M. and HanifDar, A., "Content Based image Retrieval Using Geometrical-Shape of Objects in Image", International Conference on Emerging Technologies (ICET'07), Islamabad, pp. 222 225, 12-13 November, 2007.Brown, L. D., Hua, H., and Gao, C. 2003. A widget framework for augmented interaction in SCAPE. 
[6] Pujari, J., Pushpalatha, S.N., Desai, P.D., "ContentBased Image Retrieval using Color and Shape Descriptors," International Conference on Signal and Image Processing (ICSIP), Chennai, pp. 239 - 242, 1517 December, 2010.

[7] Deselaers, T., Keysers, D. and Ney, H., "FIRE - Flexible Image Retrieval Engine: ImageCLEF 2004 Evaluation," Proceedings of the 5th conference on Cross-Language Evaluation Forum: multilingual Information Access for Text, Speech and Images, vol. 3491, pp. 688-698, 2005.

[8] Petland, A., Picard, R.W., Sclaroff, S., "Photobook: Content-Based Manipulation of Image Databases," International Journal of Computer Vision, vol. 18, pp. 233-254, June 1996.

[9] Manjunath, B.S., Salembier, P., Sikora,T. “ Introduction to MPEG-7: Multimedia Content Description Interface, vol. 1, April 2002.

[10] Anantharatnasamy, P., Sriskandaraja, K., Nandakumar, V. and Deegalla, S., "Fusion of Colour, Shape and Texture Features for Content Based Image Retrieval", $6^{\text {th }}$ International Conference on Computer Science \& Education (ICCSE), Colombo, 26-26 April, 2013, pp. $422-425$.

[11] Acharjya, P.P., Das, R., Ghoshal, D., "A Study on Image Edge Detection Using the Gradients," International Journal of Scientific and Research Publications, vol. 2, pp. 1-5, December 2012.

[12] Liu, H. and Yu, X., "Application Research of k-means Clustering Algorithm in Image Retrieval System", Proceedings of the Second Symposium International Computer Science and Computational Technology (ISCSCT '03), China, pp. 254-255, 26-26 December, 2003.

[13] Wang, J.Z., "Wang Database," (online). Available at: http://wang.ist.psu.edu/.

[14] Muller, H., Muller, W., Squire, D.M., Maillet, S.M., Pun, T., "Performance Evaluation in Content-Based Image Retrieval: Overview and Proposals," Swiss National Foundation For Scientific Research, pp. 1-15, 15 Aug, 2010.

[15] Lande, M.V., Bhanodiya, P., Jain, P., “An Effective Content-Based Image Retrieval Using Color, Texture and Shape Feature," Intelligent Computing, Networking, and Informatics, Advances in Intelligent Systems and Computing 243, vol. 243, pp. 1163-1170, 2014.

[16] Wang, X.-Y., Yu, Y.-J., Yang, H.-Y., “An effective image retrieval scheme using color, texture and shape features," Computer Standard and Interfaces, vol. 33, pp. 59-68, 2011. 